\title{
The role of surgery in chronic pancreatitis
}

\author{
Carlo Frola - Murali Somasundaram - Deepak Hariharan • Valdone Kolaityte - Shailesh Mohandas · \\ Stefan Stättner (D) V Vincent S. Yip
}

Received: 15 March 2019 / Accepted: 2 April 2019 / Published online: 7 May 2019

(c) The Author(s) 2019

\begin{abstract}
Summary
Background Chronic pancreatitis (CP) remains a complex condition resulting in significant morbidity and suffering in patients, often over a long period of time. Treatment is mostly centred on a conservative approach, with a variety of more aggressive options being trialled over the years utilising numerous endoscopic and surgical techniques.

Methods This review provides an overview of current treatment options for $\mathrm{CP}$, the literature search was performed via PubMed. Personal experiences from the authors on how to approach the disease from the surgeon's perspective are added. The outline includes pathophysiologic aspects, classifications and patientcentred surgical approaches.

Results There has not been a standardized treatment for CP so far as clinical and radiological appearance of the disease have a wide range due to great heterogeneity of this complex disease; therefore, level 1 evidence for treatment of CP remains low. More recently, different approaches to surgical management have been trialled. With personalized surgery, long-term pain relief is achievable in up to $90 \%$ with low morbidity. Autologous islet cell transplantation is a feasible option in selected patients to avoid endocrine insufficiency. Conclusion A tailored approach to CP patients is mandatory in this heterogeneous disease. Surgery provides good outcomes especially as prophylaxis for
\end{abstract}

C. Frola · M. Somasundaram · D. Hariharan · V. Kolaityte · S. Mohandas $\cdot$ V. S. Yip

Barts Hepato-Pancreato-Biliary Surgery, The Royal London Hospital, Whitechapel Rd, London, E1 1BB, UK

PD Dr. S. Stättner $(\square)$

Department of Visceral, Transplantation and

Thoracic Surgery, Medical University of Innsbruck,

Anichstraße 35, 6020 Innsbruck, Austria

stefan.staettner@i-med.ac.at and treatment of chronic pain. A multidisciplinary approach is mandatory, including physicians, pancreatic surgeons, endoscopists, dieticians and radiologists.

Keywords Pancreatitis, chronic - Pancreatectomy • Pancreaticojejunostomy - Surgical procedures, operative - Cholangiopancreatography, endoscopic retrograde

\section{Introduction}

Chronic pancreatitis (CP) remains a challenging condition to manage, despite its establishment as a clinical entity almost 80 years ago [1]. Although most studies demonstrate incidence and prevalence rates of around 7 per 100,000 and 28 per 100,000, respectively, the morbidity associated with the disease causes a disproportionate impact on healthcare systems worldwide [2-5]. Despite advances in understanding the pathophysiology, technological advances in endoscopic techniques and improvement in outcomes in pancreato-biliary surgery, management remains unstandardised with contention in approaches, of which some were more recently addressed by clinical trials $[6,7]$. These precedents may lead to difficulties for clinicians in decision making for patients with CP.

The heterogeneity of the pancreas and its multifunctionality results in the complex pathophysiology at play in CP. A series of mechanisms have been postulated as to the pathophysiology, including (i) the direct effect of toxic metabolites (e.g. tobacco, alcohol) on acinar cells, (ii) a "two-hit" model in which an episode of acute pancreatitis causes activation of pancreatic stellate cells with subsequent fibrosis, (iii) ductal dysfunction causing obstruction secondary to formation of protein plugs, (iv) oxidative stress in acinar cells secondary to free radicals promoting fusion of lysosomes and (v) a necrosis-fibrosis sequence as 
a result of repeated episodes of acute pancreatitis [8]. As demonstrated by the numerous proposed pathophysiological mechanisms, definitive understanding of the predominant process remains poor.

Pain remains the key symptom of clinical presentation and the primary focus of clinical management. It has been hypothesized that recurrent episodes of pain are due to progressive pancreatic fibrosis [9]. Delays in diagnosis from the onset of symptoms to establishment of diagnosis may range from 62 to 81 months. Following repeated attacks of pain, the pancreas may undergo full fibrosis leading to a painless state over a period of years [10]. However, not all patients with CP present with pain or develop symptoms of exocrine/endocrine insufficiency. Thus, from a clinical viewpoint, patient subgroups exist within the spectrum of CP for which different treatment combinations may be required. The challenge for the pancreatic surgeon is to identify this subgroup of CP patients who will benefit from operative intervention at the appropriate juncture.

\section{Diagnosis and classification of chronic pancreati- tis}

The presence of relevant aetiological risk factors should be sought early in clinical assessment, such as alcohol abuse, nicotine use, gallstones and family history in order to prevent delayed diagnosis. Episodes of intermittent abdominal pain likely represent repeated attacks of acute pancreatitis which may go undiagnosed. It is now widely accepted that a single episode of clinically diagnosed acute pancreatitis may represent a sentinel event which results in CP [12, 13]. In addition to these aforementioned clinical presentations, patients may be asymptomatic and detected incidentally following imaging for other reasons. The resultant mix of possible presentations causes difficulties in identifying cases, monitoring and trialling treatments.

Computed tomography (CT) remains the best initial imaging modality to diagnose $\mathrm{CP}$, with the findings of pancreatic duct dilatation, pancreatic atrophy, pancreatic mass, calcification and/or recurrent pseudocyst formation [11]. However, normal pancreatic morphology may also be observed on CT imaging with early $\mathrm{CP}$, making diagnosis particularly challenging.

It is commonly known that longstanding pre-existing $\mathrm{CP}$ is a risk factor for pancreatic cancer. Although only $1.8 \%$ of these patients will develop pancreatic cancer within 10 years from the diagnosis and $4 \%$ after 20 years [14], it may be very difficult to rule out pancreatic cancer in the presence of a pancreatic mass. There have been a number of developments in imaging and molecular technologies to aid in differentiating benign from malignant mass lesions in patients with chronic pancreatitis. While some such as EUS-FNA (endoscopic ultrasound/fine needle aspiration) and advanced CT/MRI (magnetic resonance imaging) techniques are already in clinical use, technologies like CE EUS (contrast enhanced endoscopic ultrasound), EUS elastography and digital image analysis require development of standardised protocols, consensus and operator training facilities before they can be inducted into regular clinical use [15]. The molecular techniques are still in the early stage of development. Continued research and development are required to help in the correct diagnosis of this challenging condition.

The desire to organise and classify patients with CP has led to development of multiple classification systems over the years, including the Marseille, Cambridge, Zurich and TIGAR-O systems amongst others [16-19]. More recently, the M-ANNHEIM classification has sought to unify these systems and permit aetiology and clinical severity to be included in stratification [20]. Whilst these systems have contributed greatly to the study of $\mathrm{CP}$, none of them are specifically focussed on a surgical approach. Although patients may be classified by these systems as "probable pancreatitis" (Zurich system), "moderate changes" (Cambridge system) or "M-ANNHEIM C", this does not assist the surgeon in making management decisions. With the increasing evidence to support surgery in treatment of CP, there is a need to stratify patients differently to aid the operative decision-making process [21].

\section{Timing of surgery}

It is widely accepted that the primary indication for surgery is the presence of pain. As discussed previously, however, the majority of patients will present with pain and this large patient subgroup needs further assessment. Medical management should be optimised to address the risk factors by reducing diabetic complications, diet/supplement administration for exocrine insufficiency, psychiatric support and analgesia for pain management prior to the consideration for surgical intervention using a multidisciplinary team approach. Once the aetiology of CP has been addressed, surgery can be considered under the proviso that basic assessment of fitness for operation has been met.

The traditional management approach to $\mathrm{CP}$ favoured a classical pathway of medical treatment followed by endoscopic intervention and, finally, surgery if previous treatments had failed [22]. The primary objective for surgery is to avoid a state of chronic, intractable pain which leads to a state of continual analgesic abuse and dependence. Pain specialists should be involved early and guide the use of effective analgesic control; however, once typical analgesia management has failed, techniques such as endoscopic coeliac plexus block/neurolysis can be applied and have been shown to provide effective pain relief in $50 \%$ of patients [23]. This procedure can be repeated safely but caution should be exer- 

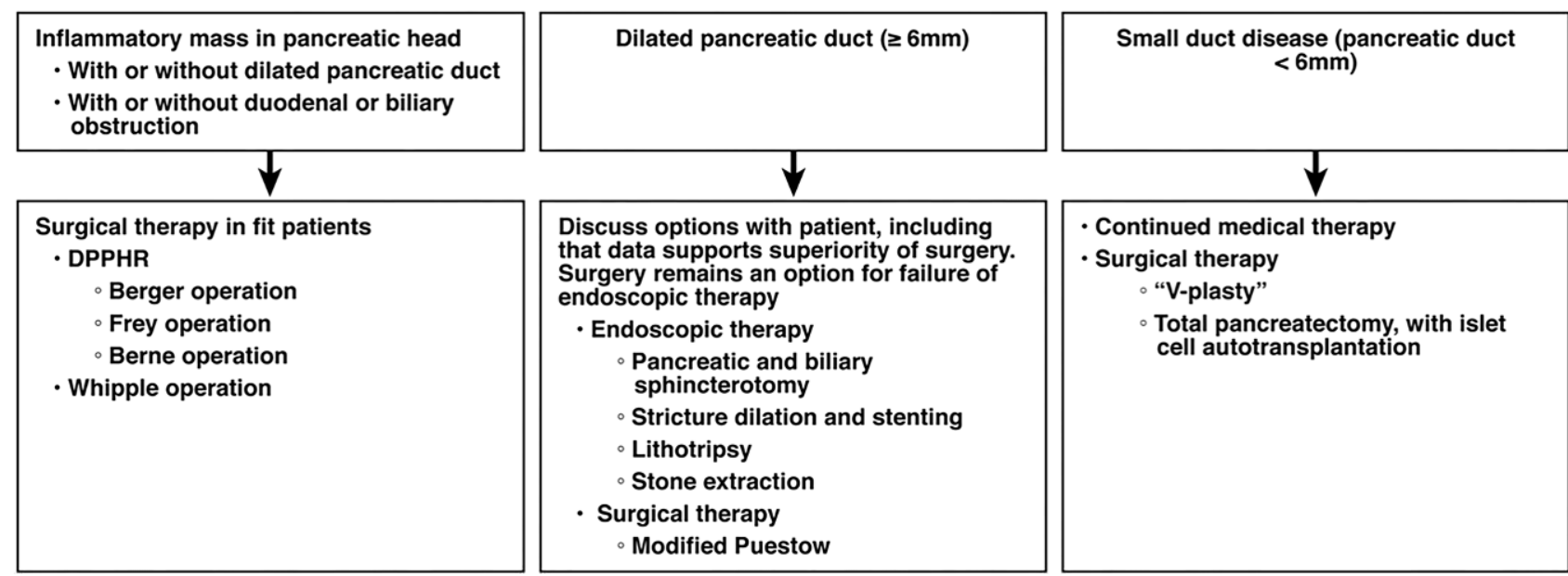

Fig. 1 Different types of CP appearance and potential treatment algorithms. DPPHR duodenum preserving pancreatic head resection

cised. Whilst patients are suffering with symptoms, the underlying cause of the pain, for example ductal obstruction secondary to calculus, would not be addressed. The result may be progressive destruction of pancreatic parenchyma and loss of function, making surgery technically more challenging, therefore worsening outcomes. It is the authors' belief that patients should not be progressed from standard analgesics to complex analgesic administration indefinitely without an overall plan for future treatment. The goal should be to avoid a state of analgesic abuse and to use such endoscopic techniques as a bridging therapy to definitive treatment [24].

Numerous endoscopic and interventional radiological techniques now exist to manage chronic pancreatitis, including endoscopic retrograde cholangiopancreatography (ERCP) and pancreatic duct stenting, endoscopic cystogastrostomy and percutaneous drainage of pseudocysts under image guidance. All these techniques play a role in patient management for chronic pancreatitis. However, in patients with chronic intractable pain secondary to $\mathrm{CP}$, evidence now supports proceeding to surgery earlier over endoscopy due to superiority in success rate, pain relief and quality of life. Surgery may need to be considered at an earlier phase than it is now, preferably within 3 years of symptomatic CP [24-27].

One of the primary problems in an endoscopybased treatment regime is that whilst treatments may be successful, their duration may be short lived, necessitating multiple procedures causing complications, which could have been avoided with a single intervention. Nevertheless, endoscopic techniques do have a role in patients who are unfit for surgery. In all other cases, they should serve as an adjunctive treatment modality in the overall patient management rather than as definitive therapy.

Moreover, early surgery may help to correct exocrine insufficiency, which commonly develops 5 to 10 years after the onset of $\mathrm{CP}$ but tends to be under- diagnosed and is under-treated in $\mathrm{CP}$ patients. $\mathrm{CP}$ may also lead to type $3 \mathrm{c}$ diabetes mellitus and early stage surgery in CP may reduce this risk [28].

\section{Surgical options}

The rationale for surgical treatment in $\mathrm{CP}$ has traditionally focused primarily on addressing the mechanical obstruction secondary to pancreatic parenchymal calcification, and its local complications secondary to pancreas parenchymal fibrosis causing biliary or duodenal obstructions, and pseudocyst formation due to recurrent inflammation and/or head of pancreas duct obstruction [29-31]. Whilst the presence of macroscopic complications plays a key role in the decision for surgical intervention, clinical symptoms must be considered in conjunction at the decision process.

Evidence thus far supports surgery over endoscopic intervention in treatment of CP and indicates that the timing of surgery should be carefully considered [24-27]. Most clinicians favour an early surgical intervention in a recent survey [32]. The subject of surgical treatment of CP has been extensively reviewed in the literature from a surgical perspective [33, 34]. However, it is still unclear regarding the optimal choice of the type of surgical technique. This variation of practice was highlighted in a recent survey of 288 pancreatologists from 47 countries. It concluded that the majority of CP management is based on local expert opinion, rather than evidence-based guidelines [32].

We hereby describe four common clinical scenarios, where most CP patients are likely to be categorized, and attempt to discuss various surgical options available for each scenario (see Fig. 1).

\section{Group 1a-inflammatory pancreatic mass with suspicious features}

Differentiation between CP and malignancy has proven to be difficult prior to surgery, especially 
Fig. 2 Schematic drawing of duodenum-preserving pancreatic head resection (DPPHR) procedures. Extent of resection is shown for Beger's operation (a) and Frey's operation (b), as well as the Berne modification (c). Panels $\mathbf{d}-\mathbf{f}$ show the corresponding surgical sites after reconstruction. (C) Republished with permission of Elsevier, from Muller et al. [35]); permission conveyed through Copyright Clearance Center, Inc. This figure is not included under the Creative Commons CC BY license of this publication

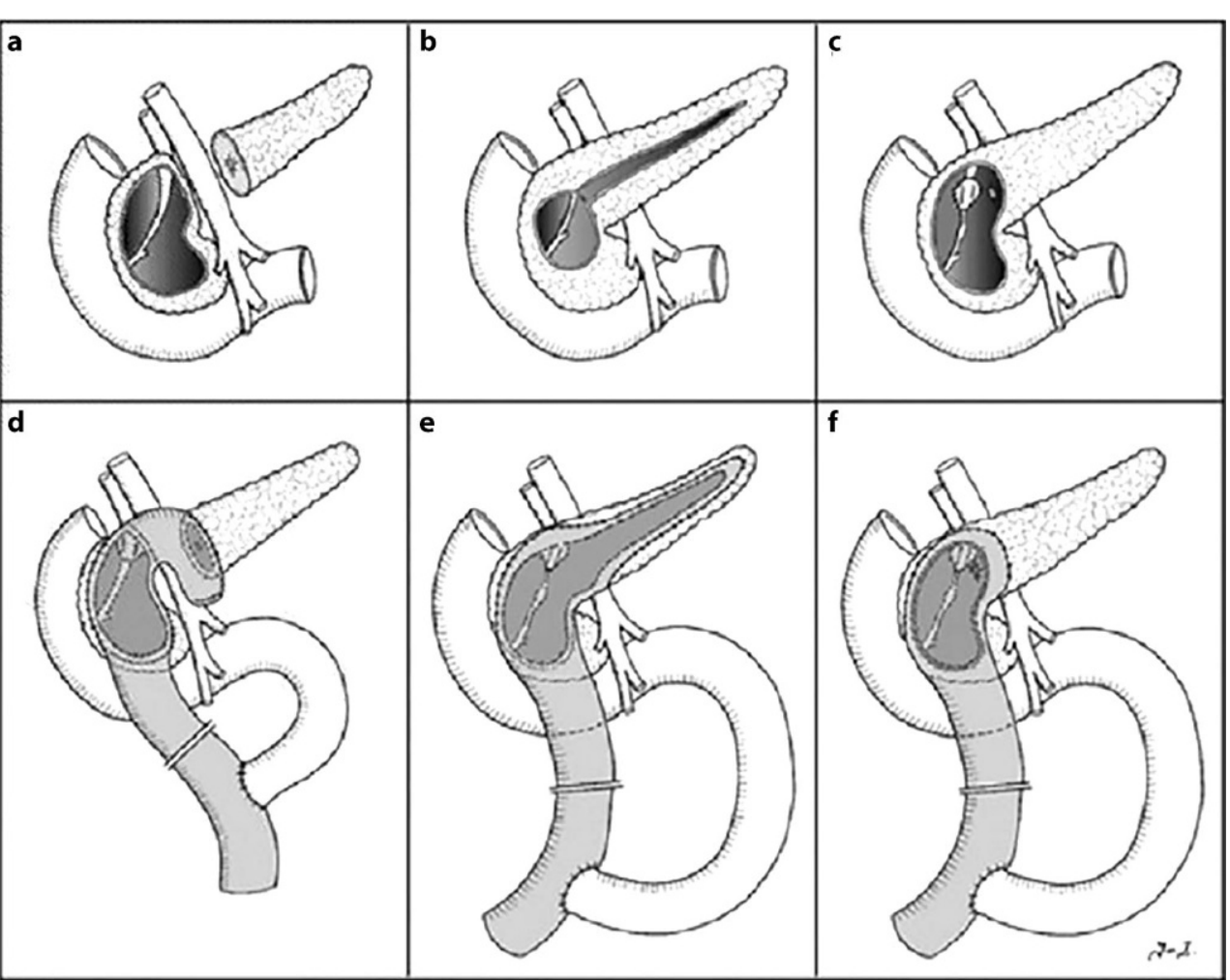

when there is a distinctive pancreatic mass identified on cross-sectional imaging [33]. Pancreatic biopsy with a frozen section should be performed at the time of surgery to establish the diagnosis of malignancy. If positive for malignancy, formal oncological resection with curative intent should be performed at the same setting.

\section{Group 1b-inflammatory pancreatic mass with benign appearance}

The identification of a pancreatic mass with benign appearance requires very careful history and examination of the recurrent inflammatory process, gradual changes of pancreas parenchyma on cross-sectional imaging over a period of time and expert radiologist interpretation. The potential false-negative diagnosis of a pancreatic mass as benign appearance has a significant impact on patient outcome.

An inflammatory mass located at the pancreatic head can cause obstruction to the pancreatic duct, bile duct and gastric outflow. The presence of such complications and intractable pain are indications to proceed to surgery, ideally prior to the commencement of opiate analgesia [32].

Notably, however, such an inflammatory mass may also be associated with chronic pain in the absence of mechanical obstruction. This is likely secondary to the postulated theory that the head of the pancreas serves as a "pacemaker", driving the process of intractable pain [34-36]. Whilst surgery has been offered to such patients, there is no body of evidence on the benefit of surgery for this particular patient group versus medical management. This is due to the small numbers of case series and the inclusion of a rather heterogeneous patient population.

Where obstruction is present, duodenum-preserving pancreatic head resection (DPPHR) and pancreaticoduodenectomy (PD) are the two most common surgical interventions. There are various techniques for DPPHR, but the most commonly performed techniques are Beger's operation, Frey's operation or Berne modification procedures. The differences in terms of their technical aspects are illustrated in Fig. 2.

Several single-centre clinical trials compared PD and DPPHR and had favoured the DPPHR approach [30, 31]. However, a multi-centre double-blinded randomised controlled superiority ChroPac trial comparing PD vs. DPPHR for chronic head pancreatitis was recently reported. The primary endpoint focused on long-term quality of life over a 24-month period following either of the two surgical interventions. The study did not demonstrate any difference in quality of life, mortality and morbidity between the interventions [6]. Based on this finding, authors therefore recommend that the choice of DPPHR or PD procedure should be based on the merit of each individual case and surgeon's preference.

\section{Group 2-isolated dilated pancreatic duct disease}

Dilatation of the pancreatic duct in CP may arise secondary to a variety of mechanisms such as ductal obstruction by calculi, ductal compression by parenchy- 
mal inflammation or mass, or localised fibrosis. If, however, the dilatation is isolated without mass in the pancreatic head, the gland can be decompressed by a drainage procedure, rather than surgical resection. This clinical scenario is best demonstrated on crosssectional imaging as the classic "chain of lakes" appearance. Drainage procedures minimise resection of any aspect of the pancreas, thereby focussing on preservation of remnant pancreatic function and minimised morbidity. However, they are contraindicated in patients with significant changes to pancreatic parenchyma where resection is normally required.

Puestow and Gillesby first described a side-to-side pancreaticojejunostomy with a distal pancreatectomy to drain the dilated pancreatic duct in patients with CP [37]. In 1960, this procedure was modified by Partington and Rochelle who performed only a side-toside pancreaticojejunostomy without a distal pancreatectomy (colloquially known as "Puestow" procedure). This procedure addresses CP in patients with a dilated pancreatic duct $(\geq 6 \mathrm{~mm})$ and no inflammatory mass in the head of the pancreas. When performed adequately, long-term pain relief has been reported in the region of $60-98 \%$, with low morbidity [38].

\section{Group 3-small pancreatic duct disease}

Small-duct CP remains rare and may be related to parenchymal gland inflammation or fibrosis causing narrowing of the pancreatic duct (PD) to $<3 \mathrm{~mm}$ [39]. Diagnosis may be difficult as typical imaging may demonstrate mild abnormalities, which can be potentially overlooked by an inexperienced radiologist. Definitive diagnosis can be sought and confirmed by secretin testing. Endoscopic ultrasound (EUS) should be considered to exclude a pancreatic ductal neoplasm. Having confirmed the diagnosis of $\mathrm{CP}$, patients should proceed to surgery for symptom control.

Some anecdotal evidence suggests that a "V-plasty" operation is the procedure of choice, with reasonable safety and median- to long-term pain relief [40-42]. However, one contraindication to offering "V-plasty" in this group of patients is the suspicion of malignancy, where an aggressive oncological resection would be much more appropriate.

\section{Group 4-atypical presentation of CP}

Cases of CP presenting differently to the above-described grouping will require an alternative/bespoke approach. A total pancreatectomy (TP) may also be offered to CP patients with endocrine insufficiency. However, the brittle diabetes resulting from TP (due to loss of glucagon as well as insulin secretion) will require careful titration of insulin administration by endocrinologists.

The long-term morbidity caused by TP can be reduced by autologous islet transplantation (TPIAT) for patients without diabetes mellitus. A meta-analysis of 12 published articles indicated that TPIAT is safe, with a 30 -day mortality of $2.1 \%$ and a long-term mortality of 1.09 per 100 person-years, which is comparable with $\mathrm{TP}$ alone. Increasing severity of pancreatic fibrosis correlates positively with poor yield of islets $(<300,000)$ and insulin dependence. C-peptide-negative diabetes, type I diabetes, portal vein thrombosis and portal hypertension/significant liver disease are considered contraindications to TPIAT [43].

Two systematic reviews which carried out metaanalyses reported pooled insulin independence rates of $27 \%$ (95\% CI: $21-33 \%$ ) and $28.4 \%$ (95\% CI: $15.7-46.0)$ at one year and 21\% (95\% CI: 16-27\%) and 19.7\% (95\% CI: 5.1-52.6\%) at two years, respectively [44, 45].

\section{Conclusion}

The complex, heterogenous nature of the pancreas results in CP manifesting in a variety of clinical presentations. Treatment of CP should follow a multidisciplinary approach involving physicians, pain specialists, endoscopists and pancreatic surgeons. Evidence now favours early surgery as the preferable treatment for patients with intractable pain over endoscopic management. Head-dominant CP has been addressed through the latest clinical trials. However, further clarity is still required for the management of non-head-dominant disease.

Acknowledgements Open access funding for this publication was granted by the Medical University of Innsbruck and the University of Innsbruck.

Funding Open access funding provided by University of Innsbruck and Medical University of Innsbruck.

Conflict of interest C. Frola, M. Somasundaram, D. Hariharan, V. Kolaityte, S. Mohandas, S. Stättner and V.S. Yip declare that they have no competing interests.

Open Access This article is distributed under the terms of the Creative Commons Attribution 4.0 International License (http://creativecommons.org/licenses/by/4.0/), which permits unrestricted use, distribution, and reproduction in any medium, provided you give appropriate credit to the original author(s) and the source, provide a link to the Creative Commons license, and indicate if changes were made.

\section{References}

1. Kloppel G. Chronic pancreatitis, pseudotumors and other tumor-like lesions. Mod Pathol. 2007;20(Suppl 1):S113-S31.

2. Levy P, Barthet M, Mollard BR, Amouretti M, MarionAudibert AM, Dyard F. Estimation of the prevalence and incidence of chronic pancreatitis and its complications. GastroenterolClin Biol. 2006;30(6-7):838-44.

3. Yadav D, Lowenfels AB. The epidemiology of pancreatitis and pancreatic cancer. Gastroenterology. 2013;144(6):1252-61.

4. Masamune A, Kikuta K, Nabeshima T, Nakano E, Hirota M, Kanno A, et al. Nationwide epidemiological survey 
of early chronic pancreatitis in Japan. J Gastroenterol. 2017;52(8):992-1000.

5. Lowenfels AB, Maisonneuve P, Grover H, Gerber E, Korsten MA, Antunes MT, et al. Racial factors and the risk of chronic pancreatitis. Am J Gastroenterol. 1999;94(3):790-4.

6. Diener MK, Huttner FJ, Kieser M, Knebel P, DorrHarim C, Distler M, et al. Partial pancreatoduodenectomy versus duodenum-preserving pancreatic head resection in chronic pancreatitis: the multicentre, randomised, controlled, double-blind ChroPac trial. Lancet. 2017;390(10099):1027-37.

7. Ali AU, Issa Y, Bruno MJ, van Goor $H$, van Santvoort $H$, Busch OR, et al. Early surgery versus optimal current step-up practice for chronic pancreatitis (ESCAPE): design and rationale of a randomized trial. BMC Gastroenterol. 2013;13:49

8. Kleeff J, Whitcomb DC, Shimosegawa T, Esposito I, Lerch MM, Gress T, et al. Chronic pancreatitis. Nat Rev Dis Primers. 2017;3:17060.

9. Pham A, Forsmark C. Chronic pancreatitis: review and update of etiology, risk factors, and management. F1000Res. 2018;7:F1000.

10. Ammann RW, Akovbiantz A, Largiader F. Pain relief in chronic pancreatitis with and without surgery. Gastroenterology. 1984;87(3):746-7.

11. Dominguez-Munoz JE, Drewes AM, Lindkvist B, Ewald N, Czako L, Rosendahl J, et al. Recommendations from the United European Gastroenterology evidence-based guidelines for the diagnosis and therapy of chronic pancreatitis. Pancreatology. 2018;18(8):847-54.

12. Kloppel G, Maillet B. The morphological basis for the evolution of acute pancreatitis into chronic pancreatitis. Virchows Arch A Pathol Anat Histopathol. 1992;420(1):1-4.

13. Ammann RW, Heitz PU, Kloppel G. Course of alcoholic chronic pancreatitis: a prospective clinicomorphological long-term study. Gastroenterology. 1996;111(1):224-31.

14. Dutta AK, Chacko A. Head mass in chronic pancreatitis: Inflammatory or malignant. World J Gastrointest Endosc. 2015;7(3):258-64.

15. Lowenfels AB, Maisonneuve P, Cavallini G, Ammann RW, Lankisch PG, Andersen JR, Dimagno EP, et al. Pancreatitis and the risk of pancreatic cancer. International Pancreatitis Study Group. N Engl J Med. 1993;328(20):1433-7.

16. Singer MV, Gyr K, Sarles H. Revised classification of pancreatitis. Report of the Second International Symposium on theClassification of Pancreatitisin Marseille, France, March 28-30, 1984. Gastroenterology. 1985;89(3):683-5.

17. Sarner M, Cotton PB. Classification of pancreatitis. Gut. 1984;25(7):756-9.

18. Etemad B, Whitcomb DC. Chronic pancreatitis: diagnosis, classification, and new genetic developments. Gastroenterology. 2001;120(3):682-707.

19. Ammann RW. A clinically based classification system for alcoholic chronic pancreatitis: summary of an international workshop on chronic pancreatitis. Pancreas. 1997;14(3):215-21.

20. Schneider A, Lohr JM, Singer MV. The M-ANNHEIM classification of chronic pancreatitis: introduction of a unifying classification system based on a review of previous classifications of the disease. J Gastroenterol. 2007;42(2):101-19.

21. Jawad ZAR, Kyriakides C, Pai M, Wadsworth C, Westaby D, Vlavianos $\mathrm{P}$, et al. Surgery remains the best option for the management of pain in patients with chronic pancreatitis: a systematic review and meta-analysis. Asian J Surg. 2017;40(3):179-85.

22. Kwon CI, Cho JH, Choi SH, Ko KH, Tirkes T, Gromski $\mathrm{MA}$, et al. Recent advances in the diagnosis and man- agement of chronic pancreatitis. Korean J Intern Med. 2019;34(2):242-60.

23. Fusaroli P, Caletti G. Is there a role for celiac plexus block for chronic pancreatitis? Endosc Int Open. 2015;3(1):E60-E2.

24. Nealon WH, Thompson JC. Progressive loss of pancreatic function in chronic pancreatitis is delayed by main pancreatic duct decompression. A longitudinal prospective analysis of the modified puestow procedure. Ann Surg. 1993;217(5):458-66. discussion66-8.

25. Dite P, Ruzicka M, Zboril V, Novotny I. A prospective, randomized trial comparing endoscopic and surgical therapy for chronic pancreatitis. Endoscopy. 2003;35(7):553-8.

26. Cahen DL, Gouma DJ, Nio Y, Rauws EA, Boermeester MA, Busch OR, et al. Endoscopic versus surgical drainage of the pancreatic duct in chronic pancreatitis. N Engl J Med. 2007;356(7):676-84.

27. Cahen DL, Gouma DJ, Laramee P, Nio Y, Rauws EA, Boermeester MA, et al. Long-term outcomes of endoscopic vs surgical drainage of the pancreatic duct in patients with chronic pancreatitis. Gastroenterology. 2011;141(5):1690-5.

28. Cui Y, Andersen DK. Diabetes and pancreatic cancer. Endocr RelatCancer. 2012;19:F9-F26.

29. Strate T, TaherpourZ, Bloechle C, Mann O, Bruhn JP, Schneider C, et al. Long-term follow-up of a randomized trial comparing the beger and frey procedures for patients suffering from chronic pancreatitis. Ann Surg. 2005;241(4):591-8.

30. Klempa I, SpatnyM, MenzelJ, BacaI, Nustede R, Stockmann $\mathrm{F}$, et al. Pancreatic function and quality of life after resection of the head of the pancreas in chronic pancreatitis. A prospective, randomized comparative study after duodenum preserving resection of the head of the pancreas versus Whipple's operation. Chirurg. 1995;66(4):350-9.

31. Farkas G, Leindler L, Daroczi M, Farkas GJr. Prospectiverandomised comparison of organ-preserving pancreatic head resection with pylorus-preserving pancreaticoduodenectomy. Langenbecks Arch Surg. 2006;391(4):338-42.

32. Issa Y, van Santvoort HC, Fockens P, Besselink MG, Bollen TL, Bruno MJ, et al. Diagnosis and treatment in chronic pancreatitis: an international survey and case vignette study. HPB (Oxford). 2017;19(11):978-85.

33. Dickerson LD, Farooq A, Bano F, Kleeff J, Baron R, Raraty $\mathrm{M}$, et al. Differentiation of autoimmune pancreatitis from pancreatic cancer remains challenging. World J Surg. 2019; https://doi.org/10.1007/s00268-019-04928-w.

34. Buchler MW, Friess H, Bittner R, Roscher R, Krautzberger W, Muller MW, et al. Duodenum-preserving pancreatic head resection: long-term results. J Gastrointest Surg. 1997;1(1):13-9.

35. Buchler MW, Friess H, Muller MW, Wheatley AM, Beger HG. Randomized trial of duodenum-preserving pancreatic head resection versus pylorus-preserving Whipple in chronic pancreatitis. Am J Surg. 1995;169(1):65-9. discussion 9-70.

36. Beger HG, Buchler M. Duodenum-preserving resection of thehead of the pancreas in chronic pancreatitis with inflammatory mass in the head. World J Surg. 1990;14(1):83-7.

37. Puestow CB, Gillesby WJ. Retrograde surgical drainage of pancreas for chronic relapsing pancreatitis. AMAArch Surg. 1958;76(6):898-907.

38. Muller MW, Friess H, Leitzbach S, Michalski CW, Berberat P, Ceyhan GO, et al. Perioperative and follow-up results after central pancreatic head resection (Berne technique) in a consecutive series of patients with chronic pancreatitis. AmJ Surg. 2008;196(3):364-72.

39. Gupta V, Toskes PP. Diagnosis and management of chronic pancreatitis. Postgrad Med J. 2005;81(958):491-7. 
40. IzbickiJR, BloechleC, Broering DC, Kuechler T, Broelsch CE. Longitudinal $\mathrm{V}$-shaped excision of the ventral pancreas for small duct disease in severe chronic pancreatitis: prospective evaluation of a new surgical procedure. Ann Surg. 1998;227(2):213-9.

41. Shrikhande SV, Kleeff J, Friess H, Buchler MW. Management of pain in small duct chronic pancreatitis. J Gastrointest Surg. 2006;10(2):227-33.

42. Yekebas EF, Bogoevski D, Honarpisheh H, Cataldegirmen G, Habermann CR, Seewald S, et al. Long-term followup in small duct chronic pancreatitis: A plea for extended drainage by "V-shaped excision" of the anterior aspect of the pancreas. Ann Surg. 2006;244(6):940-6. discussion 6-8.

43. Bellin MD, Freeman ML, Gelrud A, Slivka A, Clavel A, Humar $\mathrm{A}$, et al. Total pancreatectomy and islet autotransplantation in chronic pancreatitis: recommendations from PancreasFest. Pancreatology. 2014;14(1):27-35.

44. Dong M, Parsaik A, Erwin P, Farnell M, Murad M, Kudva Y. Systematic review and meta-analysis: islet autotransplantation after pancreatectomy for minimizing diabetes. Clin Endocrinol (Oxf). 2011;75(6):771-9.

45. Wu Q, Zhang M, Qin Y, Jiang R, Chen $\mathrm{H}, \mathrm{Xu} X$, Yang T, Jiang K, Miao Y. Systematic review and meta-analysis of islet autotransplantation after total pancreatectomy in chronic pancreatitis patients. Endocr J.2015;62(3):227-34.

Publisher's Note Springer Nature remains neutral with regard to jurisdictional claims in published maps and institutional affiliations. 